Review Article

\title{
Molecular Mechanisms Contributing Bacterial Infections to the Incidence of Various Types of Cancer
}

\author{
Salah A. Sheweita $\mathbb{D D}^{1,2}$ and Awad S. Alsamghan $\mathbb{D D}^{3}$ \\ ${ }^{1}$ Department of Biotechnology, Institute of Graduate Studies and Research, Alexandria University, Alexandria 21526, Egypt \\ ${ }^{2}$ Department of Clinical Biochemistry, Faculty of Medicine, King Khalid University, Abha, Saudi Arabia \\ ${ }^{3}$ Department of Family and Community Medicine, Faculty of Medicine, King Khalid University, Abha, Saudi Arabia
}

Correspondence should be addressed to Salah A. Sheweita; ssheweita@yahoo.com

Received 10 March 2020; Revised 11 June 2020; Accepted 17 June 2020; Published 8 July 2020

Guest Editor: Young-Su Yi

Copyright (c) 2020 Salah A. Sheweita and Awad S. Alsamghan. This is an open access article distributed under the Creative Commons Attribution License, which permits unrestricted use, distribution, and reproduction in any medium, provided the original work is properly cited.

\begin{abstract}
Cancer causes a major health concern worldwide due to high incidence and mortality rates. To accomplish this purpose, the Scopus, PubMed, and Web of Science databases were searched using the keywords bacteria and cancer. Most of published research addressed several different factors that induced cancer, such as toxins, medications, smoking, and obesity. Nonetheless, few studies are dealing with cancer induction via bacterial infection. In addition, mechanisms of cancer induction via bacterial infections are not well understood. Therefore, in this review, we will shed light on different bacteria that induced cancer via different molecular mechanisms. Among the bacterial infection that induced cancer, Helicobacter pylori was the first recognized bacteria which caused gastric cancer and might be also linked to extragastric cancer in humans. H. pylori has been associated with adenocarcinoma in the distal stomach by its ability to cause severe inflammations. It has been found that inflammations induced cancer via different mechanisms including induction of cell proliferation and production of high levels of free radicals. Recently, free radicals were found to induce and cause various types of cancer. Salmonella typhi has been found to be associated with gallbladder carcinoma (GBC). Also, intercellular infection of lungs with Chlamydia pneumoniae was found to contribute as one of the ethological factors of lung cancer. Moreover, infection of the urinary tract with Staphylococcus aureus, Klebsiella spp., and Proteus mirabilis has been found to cause bladder cancer. These microorganisms produce a high level of $\mathrm{N}$-nitrosamines which are metabolically activated leading to the generation of alkylating agents that damage DNA and other macromolecules. It is concluded that a certain bacterium is linked with induction of a specific type of cancer via different molecular and biochemical mechanisms as discussed in the text in details. This infection could potentially affect human health in different ways. In addition, it is important to know the possible factors involved in cancer induction for better treatment of cancer patients.
\end{abstract}

\section{Introduction}

Genetic, environmental, and dietary factors are identified as the main factors of cancer induction, and their interaction leads to carcinogenesis. Environmental factors such as tobacco smoke and occupational exposure to hazardous chemicals account for $90 \%$ of all cancers. The majority of the exogenous compounds are chemical carcinogens which undergo metabolic activation to form metabolites which interact with cellular macromolecules and initiate carcinogenesis by causing damage to the DNA, hence are called exogenous genotoxic carcinogens. These carcinogens consist of a wide variety of compounds, which differ in their chemical structure but possess a common ability to form chemical bonds with DNA, resulting in the generation of "DNA adducts." The formation of these DNA adducts is recognized as the initial step in chemical carcinogenesis $[1,2]$. In addition, the initial stage of gene mutilation is also based on endogenous mechanisms that cause mutations or even gene deletions. Very common endogenous mediators are free radicals or reactive oxygen species (ROS), which cause oxidative damage to DNA and cause different mutations (Figure 1) 


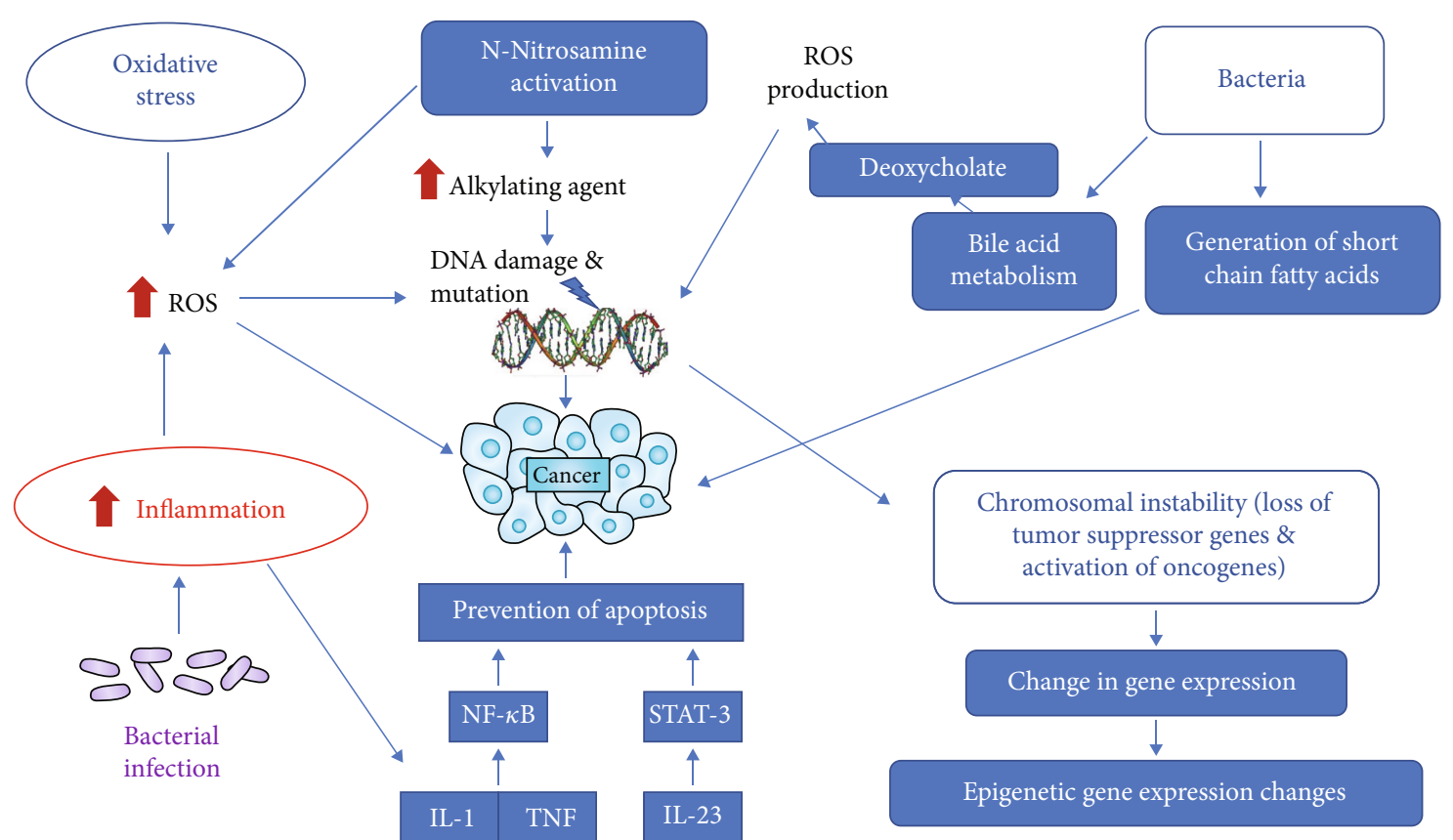

FIGURE 1: Different molecular mechanisms of carcinogenesis induced by bacterial infection and oxidative stress.

$[3,4]$. Recent studies have shown a causative relationship between bacterial infection and the onset of cancer in organs such as lungs, colon, and cervix, which are constantly exposed to bacteria (Figure 1) [5]. The harmony of cells and the control of its growth and proliferation are regulated by a well-synchronized signalling pathway. Any alteration or deregulation of these pathways triggers carcinogenesis. During bacterial infection, various bacteria target and trigger these signalling pathways. Therefore, in this review, we have focused on the role of some bacteria in the incidence of cancer since a number of bacteria have been associated with cancer via triggering signalling pathways. Helicobacter pylori, Salmonella typhi, Streptococcus bevies, and Chlamydia pneumonia cause stomach, gallbladder, colorectal, and lung cancers, respectively, through different mechanisms.

\subsection{Salmonella typhi Induced Gallbladder Cancer. Salmonella} typh $i$ is a rod-shaped gram-negative bacterium of the Enterobacteriaceae family, which is well known to cause typhoid or enteric fever. These bacteria colonize in the gallbladder causing asymptomatic chronic infection [6]. Epidemiological studies from S. typhi endemic regions have shown that most of the chronically infected carriers developed gall cholelithiasis, a primary predisposing reason for the onset of gallbladder cancer (GBC). Typhoid toxins produced by $S$. typhi have a carcinogenic potential which damages the DNA and alters the cell cycle in the infected cells. Apart from this, the extracellular polymeric substances (EPS) produced in the biofilm of $S$. typhi are the key factor for persistent infection and cholecystitis leading to exposure of the epithelium to carcinogenic toxins produced by $S$. typhi [6]. Until the 1990s, in Chile, under the backdrop of a typhoid epidemic, a high prevalence of gallbladder cancer incidence was observed, which was mainly attributed to its association with Salmonella enterica serovar typhi (S. typhi) Vi antibodies. How- ever, the exact mechanism underlying this association is still under investigation [7]. Another study in India claimed chronic Salmonella enterica serovar typhi infection to be a significant risk factor for the development of gallbladder cancer, although no direct association and mechanism have been explained yet [8].

1.1.1. Molecular Mechanism Underlying Induction of Gallbladder Cancer. The role of Salmonella typhi in altering the genomic sequence of tumor protein p53 (TP53) and amplification of protooncogene c-MYC results in malignant transformation from predisposed mice gallbladder organoids and fibroblasts (Table 1 and Figure 1). Salmonella typhi effectors released during an infection contribute to the autoactivation of protein kinase which is triggered by mitogen (MAPK) and the Akt pathways (Table 1). This process is pathognomonic in initiating as well as sustaining malignant transformation, which is a consistent observation in gallbladder cancer patients in India. Hence, the role of Salmonella typhi predisposed epithelium of gallbladder to toxic metabolites was established [8]. Another mechanism is primarily attributable to the production by $S$. typhi of genotoxic substances (cytolethal distending toxin B (CdtB)), which is the functional unit of cytolethal distending toxin (CDT) and cytotoxic necrotizing factor 1 (CNF1). The CdtB works by targeting the DNA in the human host cells while CNF1 inhibits the activity of cytokines leading to inflammation and cell cycle inhibition [9] (Table 1 and Figure 1). Moreover, CNF1 also affects the transcription termination process in prokaryotes by altering the Rho proteins [9]. S. typhi changed the protein expressions of $\mathrm{CdtB}$ and $\mathrm{CNF} 1$ leading ultimately to cancer of the gallbladder.

The function of the gallbladder is to store bile, which consists of bile salts and acids. Various mechanisms have been suggested in which altered bile salt metabolism produces 
TABLE 1: Types of cancer induction and mechanisms of carcinogenesis induced by different microbiota.

\begin{tabular}{|c|c|c|c|}
\hline Cancer & Bacteria inducing cancer & Mechanisms of carcinogenesis & References \\
\hline Gallbladder cancer & Salmonella typhi & $\begin{array}{c}\text { Changes in the sequence of p53 gene; activation } \\
\text { of protein kinase; cytolethal distending toxin B } \\
\text { (CdtB); biliary deoxycholate; cholic acid derivatives; } \\
\text { 5-alpha,6-alpha-epoxide cholesterol; upregulation } \\
\text { of the PI3K pathway }\end{array}$ & {$[8,10-13]$} \\
\hline Lung cancer & Chlamydia pneumoniae & $\begin{array}{l}\text { Alteration in apoptosis and/or cell programming } \\
\text { signalling; overexpression of miRNA-328; by } \\
\text { stimulating lung-resident } \gamma \delta \text { T cells; development } \\
\text { of Myd88-dependent IL-1b and IL-23; generation } \\
\text { of reactive oxygen species; increased secretion } \\
\text { of cytokines, IL-8, IL-10, and TNF }\end{array}$ & {$[5,19-24]$} \\
\hline Colorectal cancer & $\begin{array}{c}\text { Streptococcus bovis, Helicobacter pylori, Bacteroides } \\
\text { fragilis, Enterococcus faecalis, Clostridium septicum, } \\
\text { Fusobacterium spp., and Escherichia coli }\end{array}$ & $\begin{array}{l}\text { Secretion of Bacteroides fragilis toxin; activation of } \\
\text { NF- } \mu \text { B; expression of IL-17A, and TNF- } \alpha \text {; } \beta \text {-catenin } \\
\text { expression, induction of IL-17R, NF- } \kappa \text {, and Stat } 3 \\
\text { signals; induction of the gene expression of } \\
\text { colibactin (clbB) and Bacteroides fragilis toxin } \\
\text { (BFT), increased colonial interleukin-17, and } \\
\text { colonic epithelial DNA damage }\end{array}$ & {$[69,72-75]$} \\
\hline Breast cancer & $\begin{array}{c}\text { Methylobacterium radiotolerans, Sphingomonas } \\
\text { yanoikuyae }\end{array}$ & $\begin{array}{l}\text { Microbiota secretes bioactive metabolites including } \\
\text { estrogens, short-chain fatty acids, amino acid } \\
\text { metabolites, or secondary bile acids; dysbiosis }\end{array}$ & {$[78,79]$} \\
\hline Bladder cancer & $\begin{array}{l}\text { Staphylococcus albus hemolytic, Staphylococcus } \\
\text { aureus, Klebsiella spp., Proteus mirabilis, and E. coli }\end{array}$ & $\begin{array}{l}\text { Formation of } \mathrm{N} \text {-nitrosamines; DNA methylation; } \\
\text { reactive chemical species }\end{array}$ & [83-87] \\
\hline
\end{tabular}

carcinogenic compounds from long-term S. typhi carriers. Bacterial enzymes which were present in S. typhi work primarily on bile acids to generate high concentrations of toxic metabolites and secondary bile acids (Figure 1). These toxic metabolites lead to the pathology of the epithelium of the gallbladder. A glycosidase enzyme, $\beta$-glucuronidase, deconjugates the conjugated primary bile salts resulting in the production of high concentrations of toxic metabolites which have carcinogenic effects [9] (Table 1). These metabolites bind to DNA in human epithelial cells, exerting its mitogenic action $[10,11]$. It has also been shown that high concentrations of biliary deoxycholate, a secondary bile acid, are present at elevated levels in patients with gallbladder carcinoma [9]. Another mechanism suggested that S. typhi mutagenicity is due to its cholesterol interaction, which ultimately forms the structural basis of the gallstones. These bacteria not only transform the bile salts into secondary forms of bile but also convert cholesterol itself into carcinogenic compounds like 5-alpha,6-alpha-epoxide cholesterol, leading to pathogenic changes in epithelial cells [12] (Table 1). Another study has shown that $S$. typhi bacteria are capable of metabolizing primary bile acids into mutagenic cholic acid derivative types in the presence of bile and cholesterol substrates that cause gall balder cancer [11].

1.2. Chlamydia pneumonia and Lung Cancer. Lung cancer is closely related to chronic inflammation, but it has not completely elucidated the causes of inflammation and the basic immune mediators. Chlamydophila (formerly called Chlamydia) pneumoniae is a species of Chlamydia, an intracellular bacterium that infects the cells of the respiratory tract in humans. It is responsible for about $10 \%$ of community- acquired pneumonia and 5\% of sinusitis, pharyngitis, and bronchitis [13]. Chronic pneumocyte infection by C. pneumoniae predisposes for the development of lung cancer, which is a major public health concern due to its high incidence and mortality [13]. The existence of C. pneumoniae in 230 lung cancer cases has been found, and the lung cancer risk was increased by 1.6 in C. pneumoniae-infected patients [14]. In addition, in patients with bronchoscopy and lung cancer, an association between chronic C. pneumoniae infection and incidence of lung cancer was found [15]. It has been found that IgA antibodies were increased in lung cancer patients infected with C. pneumoniae [16]. In another study, increased C. pneumoniae-specific IgA levels in smokers with lung cancer were found [17].

1.2.1. Mechanism of Lung Cancer Induction. Post chlamydial infection, numerous proteins are released which are hypothesized to cause lung cancer by targeting either mitochondrial or cytoplasmic cellular activities. Their mechanism of action is by competitive inhibition where these targeting proteins and host proteins compete to bind with the substrate [5]. In a study done by Alshamsan et al., it was reported that out of 1112 proteins derived from C. pneumoniae, 183 and 513 proteins targeted the mitochondrial and cytoplasmic cellular processes of the host cells, respectively [18]. This leads to disturbance of normal cellular growth, resulting in an alteration in apoptosis or programmed cell death leading to the development of lung cancer. It is also assumed that these proteins are incorporated into the intracellular organelles leading to the formation of a host cell proteome. Various bioinformatics tools such as nuclear localization signal (NLS) mapper, HummPLoc 2.0, ExPASy pI/Mw tool, and balanced subcellular 
localization predictor (BaCeILo) showed that 47 of the 1112 bacterial proteins were responsible for nuclear targeting which altered the host replication and transcription [5].

Many evidences have showed that microRNA (miRNA) played an important role in the metastasis and progression of lung cancer. A miRNA, miR-328, targets H2AX (a histone protein) in the regulation of lung cancer cell apoptosis (Table 1). Overexpression of miRNA-328 is associated with lung cancer, whereas its downregulation was shown to decrease the incidence of lung cancer that is induced by $\mathrm{C}$. pneumoniae. In addition, suppression of miRNA-328 causes increased caspase 3 activity and apoptosis in cancer cells, resulting in lesser tumor volume [19].

A diverse bacterial community is colonized in the lung mucosal tissue and is commonly linked to clinical outcomes in patients with lung cancer which triggers lung adenocarcinoma-related inflammation by stimulating lungresident $\gamma \delta \mathrm{T}$ cells [20]. It has been found that commensal bacteria stimulated the development of Myd88-dependent IL-1b and IL-23 from myeloid cells, inducing the proliferation and activation of $\mathrm{Vg} 6+\mathrm{Vd} 1+\gamma \delta \mathrm{T}$ cells containing IL17 and other effector molecules to promote inflammation and proliferation of tumor cells (Figure 1) [20]. Local microbiota-immune crosstalk has been found to be correlated with the development of lung tumors, and molecular mediators that can serve as effective targets for lung cancer intervention have been identified (Table 1) [20]. Other mechanisms of bacterial infection causing lung cancer are the gene damage and the neoplastic transformation which are triggered by inflammatory mediators, nitric oxide (NO), and other reactive oxygen species (Figure 1) [21, 22]. An in vitro study has shown that chronic bacterial infection by C. pneumoniae causes increased secretion of cytokines, IL-8, IL-10, and TNF, in human alveolar macrophages and peripheral blood mononuclear cells (Table 1 and Figure 1) [23]. IL-8 is an angiogenic factor acting as a promotor of tumor growth of non-small-cell lung carcinomas in humans [24, 25]. Moreover, nitric oxide liberation is also increased in chlamydial infections which might induce an inflammatory response that leads to the development of lung cancer [26].

1.3. Helicobacter pylori and Gastric Cancer. Helicobacter pylori (H. pylori) is a gram-negative microaerophilic bacterium having a helical shape with an ability to penetrate the mucoid lining of the stomach causing infection. Although many patients infected with $H$. pylori may be asymptomatic, long-term complications include gastric ulcers, inflammation of the gastric lining leading to gastritis, and gastric carcinoma. Gastric carcinoma is a global health concern due to high morbidity and mortality. Though there are numerous predisposing risk factors such as family history, dietary habits (high salts and nitrates), alcohol, and smoking, H. pylori infection has one strongest association with gastric carcinoma [27]. Apart from environmental, dietary, and genetic factors which play a vital role in the development of most of the cancers, infection by $H$. pylori also causes cancer [28, 29]. The ability of the bacteria to enter the gastric cells and colonize for years allows it to interact with human cells and impart its harmful effects. These microbes release an onco- protein, CagA, which affects the normal epithelial cell division in the gastric mucosa (Table 1) [30]. Other factors such as environmental, dietary (essential micronutrients), and gastrointestinal microflora also increase the carcinogenic potential of $H$. pylori [30]. In order to study the association between $H$. pylori and gastric carcinoma, epidemiological studies were carried out since 1991. Numerous studies on a large number of individuals were conducted, and reports were published [31-33]. These studies provided valuable insights and strongly encouraged subsequent studies. Later, in another study, it was demonstrated that $H$. pylori antibodies were highly prevalent in asymptomatic patients who developed gastric carcinoma $[34,35]$. Nolen et al. reported an earlier onset of gastric carcinoma and a higher mortality rate in Alaska Native people as compared to the US population. This was attributed to the higher prevalence of $H$. pylori infection, antimicrobial resistance, and reinfection in people in Alaska Natives as compared to the US population [36]. A study in Japan on 213 gastric cancer patients showed $88.2 \%$ of patients having higher levels of $H$. pylori antibodies. This too suggests the role of $H$. pylori in various preceding stages such as acute gastritis, atrophic gastritis, intestinal cell metaplasia, and finally intestinal type of gastric carcinoma [37]. Another study by Frydman et al. showed a strong relationship between $H$. pylori infection and gastric cancer in younger gastric carcinoma patients [38].

\subsubsection{Mechanisms of Induction of Gastric Carcinoma.} Patients with a history of $H$. pylori infection underwent annual endoscopic observation after eradication and were screened for novel markers such as gastric epithelium released tissue, protein biomarkers, and other proteins associated with cellular metaplasia in dysplasia in carcinoma. Other markers such as CD44, a signalling molecule in cell proliferation and differentiation, and metallopeptidase, an immune response-mediated marker, were also assayed (Table 1) $[39,40]$. Metaplastic intestinal cells are characterized by overexpression of gastrointestinal stem cell markers like PROM1 gene product, leucine-rich repeat-containing G-protein coupled receptor 5 (LGR5), and genes related to the metabolism of messenger RNA and nucleic acids [39].

Various mechanisms have explained the role of $H$. pylori in altering the chemical contents of gastric juice and changes in gastric mucosal cells which cause chronic inflammation and subsequent carcinogenesis [41]. Colonization of gastric mucosal cells by $H$. pylori results in an inflammatory response by host cells. This leads to infiltration of host cells by macrophages, polymorph, nuclear leukocytes, T lymphocytes, and B lymphocytes. Therefore, the pathogen, along with its induced cytokines, stimulates the accumulation and activation of inflammatory cells [42]. Infiltration of gastric mucosal cells by activated inflammatory cells and neutrophils following infection by $H$. pylori produces free oxygen radicals both in the pyloric and duodenal mucosa which upregulates the production of IL-8 resulting in a greater inflammatory response [43-46]. These free radicals, being highly reactive, cause damage to proteins and DNA resulting in mutations (Table 1) [47]. Data showing the role of free radicals in the onset of gastric carcinoma is lacking [48]. Post H. pylori 
infection results in increased ammonia levels in the gastric mucosal cells of rats, which acted as promotors of gastric carcinoma by inducing $\mathrm{N}$-methyl-N-nitro- $\mathrm{N}$-nitroso guanidine (MNNG) [49]. Ascorbic acid, an antioxidant, reacts with nitroso compounds producing nitric oxide instead of harmful N-nitroso compounds [50]. Ascorbic acid concentration is lowered in the gastric juice following $\mathrm{H}$. pylori infection, resulting in increased activity of free intermediate radicals. However, following eradication of $H$. pylori, increased gastric juice levels of vitamin $C$ were observed [51-53]. As with most of the other microbes, the oncogenic activity in $H$. pylori is attributed to its proteins. Expression of cytotoxic mediators such as CagA and VacA by $H$. pylori causes activation or differentiation of gastric fibroblasts in rats which disrupt multiple cell signalling and proliferation pathways. Some major pathways include deregulation of Janus kinase/signal transducers, activation of nuclear factor kappa B (NF- $\kappa \mathrm{B})$, and activation of transcription (JAK/STAT), which lead to inflammation and initiation of carcinogenic cascades (Table 1) [54]. Although the inflammatory process begins in the epithelial cells, it spreads to the surrounding activated fibroblast cells resulting in tumor progression, invasion, and metastasis. The expression of downstream targets of STAT3 and the epithelial-mesenchymal transition inducing transcription factor (EMT-TFs) are increased in activated fibroblasts (Figure 1) [54, 55].

AMP-activated alpha 1 catalytic subunit (PRKAA1) is one of the subunits of the mammalian $5^{\prime}$-AMP-activated protein kinase (AMPK). They play a crucial role in the maintenance of intracellular energy metabolism and hence are considered as a gastric carcinoma risk factor [56]. In NF$\kappa$ Bp50 knockdown rats, $H$. pylori infection upregulates the expression of $\mathrm{p}-\mathrm{NF}-\kappa \mathrm{Bp} 50, \mathrm{NF}-\kappa \mathrm{Bp} 50$, and PRKAA1 expression, which promotes carcinogenesis. PRKAA1 knockdown in gastric cancer cells showed a significant decrease in cell invasion and migration. It also inhibited the expression of MMP-2 and activation of NF- $\kappa \mathrm{B}$, whereas on the contrary, PRKAA1 involved in NF- $\kappa$ Bp50 mediated gastric cancer cell invasion and migration indicated their role in gastric cell carcinogenesis [56]. H. pylori-induced inflammatory response of gastric cells leads to increased epithelial cell turnover by increasing its proliferation and apoptosis. Apart from $H$. pylori, other inflammatory markers such as TNF and interferon-gamma (INF- $\gamma$ ) also trigger apoptosis. Another marker identified as $H$. pylori activated peripheral blood mononuclear cells (PBMCs) upregulates the expression of Fas antigen in RGM-1 (Rat Gastric Mucosal Cell First) gastric cells. In the presence of Fas ligand, RGM-1 cells and PBMC medium showed immense and rapid cell proliferation and cell death (Table 1) $[29,57,58]$.

Increased gastric mucosal cell turnover also increases the demand for a DNA repair system. Increased cell proliferation results in increased rates of mutation, hence requiring greater surveillance and rectification by DNA mismatch repair (MMR) [59]. Therefore, decreased MMR activity results in mutation. Microsatellite instability (MSI) is a marker of deficiency of DNA MMR activity (Figure 1). Mutations in hMSH3 and hMSH6 (DNA MMR gene), receptors of growth factors, and transforming growth factor $\beta$-RII are seen in
MSI-positive gastric carcinoma [60-62]. Other DNA MMR gene, hMLH1, and sometimes hMSH2 expression are completely lost [63-67]. These findings are enough to suggest that $H$. pylori causes deficient MMR in the gastric mucosal cells, resulting in the development of early stages of gastric carcinoma (Table 1).

1.4. Colorectal Cancer and Bacterial Infection. For more than 100 years ago, bacteria were first identified in human tumors. However, the classification of the tumor microbiome remained difficult due to its low abundance [68]. Each type of tumor has a distinct composition of microbiome [68]. The symbiotic relationships between resident microorganisms and the digestive tract contribute significantly to the maintenance of gut homeostasis [69]. Changes to the microbiota triggered by changes in the environment (e.g., infection, diet, and/or lifestyle) may, however, disrupt this symbiotic relationship and facilitate diseases such as inflammatory bowel diseases and cancer. Colorectal cancer is a complex mixture of tumor cells, nonneoplastic cells, and a significant number of microorganisms, and microbiota involvement in colorectal carcinogenesis is becoming increasingly apparent. Nevertheless, several changes in gut microbiota's bacterial composition have been documented in colorectal cancer, indicating a major role for dysbiosis in colorectal carcinogenesis [69]. Some bacterial species, such as Streptococcus bovis, Helicobacter pylori, Bacteroides fragilis, Enterococcus faecalis, Clostridium septicum, Fusobacterium spp., and Escherichia coli, have been identified and suspected to play a role in colorectal carcinogenesis (Table 1) [69]. The potential interactions between bacterial microbiota and colorectal carcinogenesis such as genotoxicity and inflammation derived from bacteria have been found [69].

A microbial etiology for colorectal human cancer (hCRC) has been suggested and pursued for a long time [70]. Establishing how one or more members of the microbiota initiate and/or promote hCRC could stimulate the development of novel prevention approaches, since hCRC has a long time to go from initiation to presentation. It has also been proposed that various intestinal microbes may lead to a common pathway to tumorigenesis [70]. Over 90 percent of hCRC is sporadic, with a small proportion of inherited mutations. Germline mutations in the tumor suppressor gene of adenomatous polyposis coli (APC) are responsible for the family adenomatous polyposis (FAP) (Figure 1) [71]. In addition, at least 80 percent of intermittent hCRC shows adenomatous polyposis coli (APC) mutations as well.

\subsubsection{Molecular Mechanism Underlying Induction of} Colorectal Cancer. Enterotoxigenic Bacteroides fragilis (ETBF) is a commensal bacterium of the human intestine and a potent initiator of colitis through the secretion of Bacteroides fragilis toxin (BFT) [72] (Table 1). BFT induces the cleavage of E-cadherin in colon cells, which then leads to the activation of NF- $\mu \mathrm{B}$. Zerumbone, a key component of the plant Zingiber zerumbet (L.), has antibacterial and anti-inflammatory effects. Treatment with zerumbone significantly reduced expression of IL-17A, TNF- $\alpha$, and KC in ETBF-infected mouse colonic tissues [72] (Table 1). Zerumbone-treated ETBF-infected 
mice also showed a decline in colon NF- $\kappa \mathrm{B}$ signalling. Moreover, HT29/C1 colonic epithelial cells treated with BFTinduced BFT signalling and IL-8 secretion. However, an Ecadherin cleavage mediated by BFT was unaffected [72]. It has been found that ETBF colonization in mice did not change after treatment with zerumbone, whereas it decreased ETBF-induced colitis through inhibition of NF- $\kappa \mathrm{B}$ signalling [72]. ApcMin mice colonized with the enterotoxigenic human pathobiont Bacteroides fragilis (ETBF) as a model of colon tumorigenesis induced by microbes have been used [73]. Bacteroides fragilis toxin (BFT) activates a procarcinogenic, multistage inflammatory cascade in colonic epithelial cells (CECs) that includes IL-17R, NF- $\kappa$ B, and Stat 3 signals (Figure 1) [73]. While necessary, activation of Stat 3 in CECs is not sufficient to cause tumorigenesis of the ETBF colon. Therefore, BFT induces a procarcinogenic signalling relay from the CEC to a Th17 mucosal response resulting in selective NF- $\kappa$ B activation in distal colon CECs, which collectively activates distal colon tumorigenesis based on myeloid cells [73].

While $\beta$-catenin signalling is documented to be associated with inflammatory responses, and BFT is known to cleave E-cadherin associated with $\beta$-catenin, little is known about inflammation control in ETBF infection by $\beta$-catenin mediation [74]. After stimulation with BFT, expression of $\beta$-catenin in intestinal epithelial cells was reduced relatively early and then recovered relatively late after stimulation to normal levels. In comparison, phosphorylation of $\beta$-catenin occurred early in stimulation at high rates in BFT-exposed cells and decreased as time went by [74] (Table 1). Inactivation of $\beta$-catenin in BFT-stimulated cells has resulted in increased NF- $\kappa \mathrm{B}$ activity and interleukin- 8 (IL-8) expression (Figure 1). In addition, glycogen synthase kinase $3 \beta$ inhibition was associated with increased $\beta$-catenin expression and attenuated NF- $\kappa \mathrm{B}$ activity and expression of IL- 8 in BFTexposed cells. These findings indicate negative control of $\beta$ catenin in BFT-stimulated intestinal epithelial cells as a consequence of acute inflammation in ETBF infection [74].

Colonic mucosa has been observed in patients with family adenomatous polyposis (FAP), who develop benign precursor lesions (polyps) early in life [75]. Patchy bacterial biofilms predominantly composed of Escherichia coli and Bacteroides fragilis were identified. Genes for colibactin (clbB) and Bacteroides fragilis toxin (BFT), which encode secreted oncotoxins, have been highly enriched in the colonic mucosa of patients with FAP compared to healthy people [75]. It has been found that a tumor colonized with E. coli (colibactin) and enterotoxigenic B. fragilis has demonstrated increased colonial interleukin-17 and colonic epithelial DNA damage with faster tumor initiation and increased mortality compared to mice with a bacterial strain alone (Table 1 and Figure 1). This study indicated an unlikely link between the early colon neoplasia and tumorigenic bacteria [75].

1.5. Breast Cancer and Bacterial Infection. The most common cancer among women is breast cancer [76]. Breast cancer is a major cause of death among women all over the world. Breast cancer has a lifetime effect on one in eight women. The number of newly diagnosed cases of invasive breast cancer in the US is estimated at 268,000 in 2019, while the newly diagnosed cases in situ are estimated at about 62,930 [77]. Of these, 41,760 women are expected to die of breast cancer in the US in 2019 [77]. In developed countries, breast cancer survival for five years is over 80 percent thanks to screening services and the consequent early detection [78].

\subsubsection{Molecular Mechanism Underlying Induction of Breast} Cancer. Breast cancer is characterized by dysbiosis, an aberrant composition of the microbiome [79]. In this study, we address differences in the metabolism of breast cancer cells, as well as breast and gut microbiome composition in breast cancer. The role of the breast microbiome in breast cancer is unclear, but the gut microbiome does seem to play a part in the disease pathology. The gut microbiota secretes bioactive metabolites that modulate breast cancer (reactivated estrogens, short-chain fatty acids, amino acid metabolites, or secondary bile acids) (Figure 1) [79]. Such blood-borne microbial metabolites have been shown to modulate breast cancer behavior. These metabolites mimic human hormones, since they are formed in a "gland" (in this case, the microbiome) and are then transferred through the bloodstream to distant sites of action. These metabolites tend to be essential tumor microenvironmental constituents [79].

While there are proven risk factors for diet, age, and genetic predisposition, most breast cancers have unknown etiologies. The human microbiota is a group of microbes that inhabit the human body. Microbial imbalance, or microbial dysbiosis, has been involved in numerous human diseases including obesity, diabetes, and colon cancer [80]. In a qualitative breast microbiota DNA study, the bacterium Methylobacterium radiotolerans has been found to be relatively enriched in tumor tissue, while the Sphingomonas yanoikuyae bacterium is relatively enriched in paired normal tissue (Table 1). In paired normal breast tissue, but not in tumor tissue, the relative abundances of these two bacterial species were inversely correlated, indicating that dysbiosis is associated with breast cancer. In addition, total bacterial DNA load was decreased in the tumor versus paired normal and healthy breast tissues. Interestingly, the bacterial DNA load was associated inversely with advanced disease, a result that may have broad implications in breast cancer diagnosis and staging. Microbial DNA is present in the breast and can affect the local immune system [80].

1.6. Bladder Cancer and Bacterial Infection. Numerous laboratory, clinical, and community-based epidemiological studies have been conducted to determine the connection between urinary tract bacterial infection and bladder carcinoma incidence. Increased risk of bladder cancer following bacterial urinary tract infection has been identified in patients with recurrent or chronic cystitis and paraplegic patients [81]. Bacteria that are present in the urine have the ability to reduce ingested nitrates into nitrite which transforms into a nitrosating agent in acidic or neutral $\mathrm{pH}$. About 39 to $66 \%$ of patients hospitalized with bladder carcinoma tested positive for bacteriuria, thus indicating urinary tract infection (UTI) [82]. In another study, urine samples were collected from 76 bladder carcinoma patients, and bacterial counts were $10^{3} \mathrm{CFU} / \mathrm{ml}$ in $60 \%$ of patients which was much 
higher than female patients. Microbial urine profile revealed the presence of Staphylococcus albus hemolytic, Staphylococcus aureus, Klebsiella spp., Proteus mirabilis, and E. coli [83].

1.6.1. Mechanism of Induction of Bladder Cancer. These species are bacteria-producing nitrate and thus play an important role in the production of N-nitrosamines (Figure 1). These organisms have been shown in vitro to perform a nitrosation reaction between ingested or metabolically derived nitrates and secondary amines under physiological $\mathrm{pH}$, leading to the formation of $\mathrm{N}$-nitrosamines (Figure 1) [84]. The formation of endogenous $\mathrm{N}$-nitrosamines leads to the initiation of neoplastic events in patients. Moreover, elevated levels of $\mathrm{N}$-nitrosamines have been consistently detected in bladder carcinoma patients [85]. Bacteriainfected rats have shown also increased nitrosation of amine precursors leading to increased levels of N-nitrosamines [85]. The presence of these compounds in urine may therefore provide the origin of initiating events crucial to the development of bladder cancer (Table 1). However, in order to communicate their carcinogenic effects, these compounds need activation to produce the reactive chemical species that can alkylate constituents of tissue. DNA methylation has been identified exclusively in patients with bladder cancer in different tissues of the human population $[86,87]$.

1.7. Conclusion. It is concluded that various specific species of bacteria have the pathogenic ability to induce carcinogenesis. Although there are some common mechanisms like the release of free radicals that cause damage to DNA and other regulatory proteins, there are other complex molecular mechanisms showing the role of bacterial proteins in the activation of specific inflammatory proteins. Therefore, in this review, we have highlighted the role of bacteria in the induction of malignancy providing evidences of their mechanism. Strong evidence from the literature showed an association of Salmonella typhi, Chlamydia pneumonia, and H. pylori with gallbladder cancer, lung cancer, and gastric cancer, respectively. Therefore, it is increasingly apparent that dissection of the complex interplay between man and microbial flora is essential to understand the pathogenesis of many malignancies.

\section{Conflicts of Interest}

The authors declare that there is no conflict of interest regarding the publication of this article.

\section{References}

[1] M. C. Poirier, R. M. Santella, and A. Weston, "Carcinogen macromolecular adducts and their measurement," Carcinogenesis, vol. 21, no. 3, pp. 353-359, 2000.

[2] S. A. Sheweita, "Drug-metabolizing enzymes: mechanisms and functions," Current Drug Metabolism, vol. 1, no. 2, pp. 107132, 2000, Review.

[3] M. H. Mostafa, S. A. Sheweita, and P. J. O'Connor, "Relationship between Schistosomiasis and Bladder Cancer," Clinical Microbiology Reviews, vol. 12, no. 1, pp. 97-111, 1999.
[4] S. A. Sheweita and A. K. Tilmisany, "Cancer and phase II drugmetabolizing enzymes," Current Drug Metabolism, vol. 4, no. 1, pp. 45-58, 2003.

[5] S. Khan, A. Imran, A. A. Khan, M. Abul Kalam, and A. Alshamsan, "Systems biology approaches for the prediction of possible role of Chlamydia pneumoniae proteins in the etiology of lung cancer," PLoS One, vol. 11, no. 2, article e0148530, 2016.

[6] E. G. Di Domenico, I. Cavallo, M. Pontone, L. Toma, and F. Ensoli, "Biofilm producing Salmonella typhi: chronic colonization and development of gallbladder cancer," International Journal of Molecular Sciences, vol. 18, no. 9, p. 1887, 2017.

[7] J. Koshiol, A. Wozniak, P. Cook et al., "Salmonella enterica serovar typhi and gallbladder cancer: a case-control study and meta-analysis," Cancer Medicine, vol. 5, no. 11, pp. 3310-3335, 2016.

[8] T. Scanu, R. M. Spaapen, J. M. Bakker et al., "Salmonella Manipulation of Host Signaling Pathways Provokes Cellular Transformation Associated with Gallbladder Carcinoma," Cell Host \& Microbe, vol. 17, no. 6, pp. 763-774, 2015.

[9] A. Sharma, K. L. Sharma, A. Gupta, A. Yadav, and A. Kumar, "Gallbladder cancer epidemiology, pathogenesis and molecular genetics: Recent update," World Journal of Gastroenterology, vol. 23, no. 22, pp. 3978-3998, 2017.

[10] Q. Shi, G. R. Haenen, L. Maas et al., "Inflammation-associated extracellular $\beta$-glucuronidase alters cellular responses to the chemical carcinogen benzo [a]pyrene," Archives of Toxicology, vol. 90, no. 9, pp. 2261-2273, 2016.

[11] C. D. Klaassen and J. Y. Cui, "Review: mechanisms of how the intestinal microbiota alters the effects of drugs and bile acids," Drug Metabolism and Disposition, vol. 43, no. 10, pp. 15051521, 2015.

[12] A. Sevanian and A. R. Peterson, "Cholesterol epoxide is a direct-acting mutagen," Proceedings of the National Academy of Sciences of the United States of America, vol. 81, no. 13, pp. 4198-4202, 1984.

[13] J.-C. J. Tsay, B. G. Wu, M. H. Badri et al., "Airway microbiota is associated with upregulation of the PI3K pathway in lung cancer," American Journal of Respiratory and Critical Care Medicine, vol. 198, no. 9, pp. 1188-1198, 2018.

[14] A. L. Laurila, T. Anttila, E. Läärä et al., "Serological evidence of an association between Chlamydia pneumoniae infection and lung cancer," International Journal of Cancer, vol. 74, no. 1, pp. 31-34, 1997.

[15] E. Brandén, J. Gnarpe, G. Hillerdal et al., "Detection of Chlamydia pneumoniae on cytospin preparations from bronchoalveolar lavage in COPD patients and in lung tissue from advanced emphysema," International Journal of Chronic Obstructive Pulmonary Disease, vol. 2, no. 4, pp. 643-650, 2007.

[16] H. Koyi, E. Branden, J. Gnarpe, H. Gnarpe, and B. Steen, “An association between chronic infection with Chlamydia pneumoniae and lung cancer. A prospective 2-year studyNote," APMIS, vol. 109, no. 9, pp. 572-580, 2001.

[17] M. Paldanius, A. Bloigu, M. Leinonen, and P. Saikku, "Measurement of chlamydia pneumoniae-specific immunoglobulin A (IgA) antibodies by the microimmunofluorescence (MIF) method: comparison of seven fluorescein-labeled anti-human IgA conjugates in an in-house MIF test using one commercial MIF and one enzyme immunoassay kit," Clinical Diagnostic Laboratory Immunology, vol. 10, no. 1, pp. 8-12, 2003. 
[18] A. Alshamsan, S. Khan, A. Imran, I. A. Aljuffali, and K. Alsaleh, "Prediction of Chlamydia pneumoniae protein localization in host mitochondria and cytoplasm and possible involvements in lung cancer etiology: a computational approach," Saudi Pharmaceutical Journal, vol. 25, no. 8, pp. 1151-1157, 2017.

[19] M. Shen, L. Cai, K. Jiang, W. Xu, Y. Chen, and Z. Xu, "The therapeutic role of inhibition of miR-328 on pulmonary carcinoma induced by chlamydia pneumoniae through targeting histone H2AX," Cancer Biomarkers, vol. 1, pp. 1-8, 2018.

[20] C. Jin, G. K. Lagoudas, C. Zhao et al., "Commensal microbiota promote lung cancer development via $\gamma \delta \mathrm{T}$ cells," Cell, vol. 176, no. 5, pp. 998-1013.e16, 2019.

[21] N. Parikh, R. L. Shuck, M. Gagea, L. Shen, and L. A. Donehower, "Enhanced inflammation and attenuated tumor suppressor pathways are associated with oncogene-induced lung tumors in aged mice," Aging Cell, vol. 17, no. 1, article e12691, 2018.

[22] G. A. de Oliveira, R. Y. S. Cheng, L. A. Ridnour et al., "Inducible nitric oxide synthase in the carcinogenesis of gastrointestinal cancers," Antioxidants \& Redox Signaling, vol. 26, no. 18, pp. 1059-1077, 2017.

[23] J. Rupp, L. Pfleiderer, C. Jugert et al., "Chlamydia pneumoniae hides inside apoptotic neutrophils to silently infect and propagate in macrophages," PLoS One, vol. 4, no. 6, article e6020, 2009.

[24] R. K. Singh, M. Gutman, R. Radinsky, C. D. Bucana, and I. J. Fidler, "Expression of interleukin 8 correlates with the metastatic potential of human melanoma cells in nude mice," Cancer Research, vol. 54, no. 12, pp. 3242-3247, 1994.

[25] M. N. Khan, B. Wang, J. Wei et al., "CXCR1/2 antagonism with CXCL8/Interleukin-8 analogue CXCL8(372)K11R/G31P restricts lung cancer growth by inhibiting tumor cell proliferation and suppressing angiogenesis," Oncotarget, vol. 6, no. 25, pp. 21315-21327, 2015.

[26] J. M. Mayer, M. L. Woods, Z. Vavrin, and J. B. Hibbs Jr., "Gamma interferon induced nitric oxide production reduces Chlamydia trachomatis infectivity in McCoy cells," Infection and Immunity, vol. 61, no. 2, pp. 491-497, 1993.

[27] Y. J. Choi and N. Kim, "Gastric cancer and family history," The Korean Journal of Internal Medicine, vol. 31, no. 6, pp. 10421053, 2016.

[28] Y. H. Park and N. Kim, "Review of atrophic gastritis and intestinal metaplasia as a premalignant lesion of gastric cancer," Journal of Cancer Prevention, vol. 20, no. 1, pp. 25-40, 2015.

[29] T. A. Sebrell, M. Hashimi, B. Sidar et al., "A novel gastric spheroid co-culture model reveals chemokine-dependent recruitment of human dendritic cells to the gastric epithelium," Cellular and Molecular Gastroenterology and Hepatology, vol. 8, no. 1, pp. 157-171.e3, 2019.

[30] M. Amieva and R. M. Peek Jr., "Pathobiology of Helicobacter pylori -Induced Gastric Cancer," Gastroenterology, vol. 150, no. 1, pp. 64-78, 2016.

[31] A. Takahashi-Kanemitsu, C. T. Knight, and M. Hatakeyama, "Molecular anatomy and pathogenic actions of Helicobacter pylori CagA that underpin gastric carcinogenesis," Cellular \& Molecular Immunology, vol. 17, no. 1, pp. 50-63, 2020.

[32] J. Y. Park, D. Forman, L. A. Waskito, Y. Yamaoka, and J. E. Crabtree, "Epidemiology of Helicobacter pylori and CagAPositive infections and global variations in gastric cancer," Toxins, vol. 10, no. 4, p. 163, 2018.
[33] A. Sokic-Milutinovic, T. Alempijevic, and T. Milosavljevic, "Role of Helicobacter pylori infection in gastric carcinogenesis: current knowledge and future directions," World Journal of Gastroenterology, vol. 21, no. 41, pp. 11654-11672, 2015.

[34] M. McClain, A. C. Beckett, and T. L. Cover, "Helicobacter pylori vacuolating toxin and gastric cancer," Toxins, vol. 9, no. 10, p. 316, 2017.

[35] J. Khatoon, R. P. Rai, and K. N. Prasad, "Role of Helicobacter pylori in gastric cancer: updates," World Journal of Gastrointestinal Oncology, vol. 8, no. 2, pp. 147-158, 2016.

[36] L. D. Nolen, S. M. Vindigni, J. Parsonnet et al., "Combating Gastric Cancer in Alaska Native People: An Expert and Community Symposium," Gastroenterology, vol. 158, no. 5, pp. 1197-1201, 2020.

[37] O. Toyoshima, T. Nishizawa, M. Arita et al., "Helicobacter pyloriinfection in subjects negative for high titer serum antibody," World Journal of Gastroenterology, vol. 24, no. 13, pp. 1419-1428, 2018.

[38] G. H. Frydman, N. Davis, P. L. Beck, and J. G. Fox, "Helicobacter pyloriEradication in patients with immune Thrombocytopenic purpura: a review and the role of biogeography," Helicobacter, vol. 20, no. 4, pp. 239-251, 2015.

[39] S. E. Morales-Guerrero, C. I. Rivas-Ortiz, S. Ponce de LeónRosales et al., "Translation of gastric disease progression at gene level expression," Journal of Cancer, vol. 11, no. 2, pp. 520-532, 2020.

[40] J. Matsuzaki, H. Tsugawa, and H. Suzuki, "Precision medicine approaches to prevent gastric cancer," Gut and Liver, pp. 1-9, 2020.

[41] H. Mei and H. Tu, "Vitamin C and Helicobacter pylori infection: current knowledge and future prospects," Frontiers in Physiology, vol. 9, 2018.

[42] A. M. O'Hara, A. Bhattacharyya, J. Bai et al., "Tumor necrosis factor (TNF)- $\alpha$-induced IL- 8 expression in gastric epithelial cells: Role of reactive oxygen species and AP endonuclease1/redox factor (Ref)-1," Cytokine, vol. 46, no. 3, pp. 359-369, 2009.

[43] G. R. Davies, C. E. Collins, N. Banatvala et al., "A direct relationship between infective load of Helicobacter pylori and oxygen free radical production in antral mucosal biopsies," Gut, vol. 34, Supplement 1, pp. 73-79, 1993.

[44] A. P. Gobert and K. T. Wilson, "Polyamine- and NADPHdependent generation of ROS during Helicobacter pylori infection: A blessing in disguise," Free Radical Biology \& Medicine, vol. 105, pp. 16-27, 2017.

[45] O. Handa, Y. Naito, and T. Yoshikawa, "Redox biology and gastric carcinogenesis: the role ofHelicobacter pylori," Redox Report, vol. 16, no. 1, pp. 1-7, 2013.

[46] S. Kyung, J. W. Lim, and H. Kim, “ $\alpha$-Lipoic acid inhibits IL-8 expression by activating Nrf 2 signaling in Helicobacter pylori-infected gastric epithelial cells," Nutrients, vol. 11, no. 10, p. 2524, 2019.

[47] D. Kidane, W. J. Chae, J. Czochor et al., "Interplay between DNA repair and inflammation, and the link to cancer," Critical Reviews in Biochemistry and Molecular Biology, vol. 49, no. 2, pp. 116-139, 2013.

[48] S. A. Sheweita and M. H. Mostafa, " $N$-Nitrosamines and their effects on the level of glutathione, glutathione reductase and glutathione $S$-transferase activities in the liver of male mice," Cancer Letters, vol. 99, no. 1, pp. 29-34, 1996. 
[49] M. Tsujii, S. Kawano, S. Tsuji et al., "Ammonia: a possible promotor in Helicobacter pylori -related gastric carcinogenesis," Cancer Letters, vol. 65, no. 1, pp. 15-18, 1992.

[50] C. P. M. S. Oliveira, P. Kassab, F. P. Lopasso et al., "Protective effect of ascorbic acid in experimental gastric cancer: reduction of oxidative stress," World Journal of Gastroenterology, vol. 9, no. 3, pp. 446-448, 2003.

[51] M. Jarosz, J. Dzieniszewski, E. Dabrowska-Ufniarz, M. Wartanowicz, S. Ziemlanski, and P. I. Reed, "Effects of high dose vitamin $\mathrm{C}$ treatment on Helicobacter pylori infection and total vitamin C concentration in gastric juice," European Journal of Cancer Prevention, vol. 7, no. 6, pp. 449-454, 1998.

[52] M. Epplein, X. O. Shu, Y. B. Xiang et al., "Fruit and vegetable consumption and risk of distal gastric cancer in the Shanghai Women's and Men's Health studies," American Journal of Epidemiology, vol. 172, no. 4, pp. 397-406, 2010.

[53] Z. W. Zhang, S. E. Patchett, D. Perrett, P. H. Katelaris, P. Domizio, and M. J. G. Farthing, "The relation between gastric vitamin $\mathrm{C}$ concentrations, mucosal histology, and CagA seropositivity in the human stomach," Gut, vol. 43, no. 3, pp. 322-326, 1998.

[54] G. Krzysiek-Maczka, A. Targosz, U. Szczyrk, M. Strzalka, T. Brzozowski, and A. Ptak-Belowska, "Involvement of epithelial-mesenchymal transition-inducing transcription factors in the mechanism of Helicobacter pylori-induced fibroblasts activation," Journal of Physiology and Pharmacology, vol. 70, no. 5, 2019.

[55] F. Teresa, N. Serra, G. Capra et al., "Helicobacter pylori and Epstein-Barr Virus Infection in Gastric Diseases: Correlation with IL-10 and IL1RN Polymorphism," Journal of Oncology, vol. 2019, Article ID 1785132, 8 pages, 2019.

[56] Y. Zhang, X. Zhou, Q. Zhang, Y. Zhang, X. Wang, and L. Cheng, "Involvement of NF- $\kappa \mathrm{B}$ signaling pathway in the regulation of PRKAA1-mediated tumorigenesis in gastric cancer," Artificial Cells, Nanomedicine, and Biotechnology, vol. 47, no. 1, pp. 3677-3686, 2019.

[57] J. E. Crabtree, "Role of cytokines in pathogenesis of Helicobacter pylori-induced mucosal damage," Digestive Diseases and Sciences, vol. 43, 9 Supplement, pp. 46S-55S, 1998.

[58] J. Houghton, L. S. Macera-Bloch, L. Harrison, K. H. Kim, and R. M. Korah, "Tumor necrosis factor alpha and interleukin $1 \beta$ up-regulate gastric mucosal fas antigen expression inHelicobacter pylori infection," Infection and Immunity, vol. 68, no. 3, pp. 1189-1195, 2000.

[59] T. A. Kunkel and D. A. Erie, "Eukaryotic Mismatch Repair in Relation to DNA Replication," Annual Review of Genetics, vol. 49, no. 1, pp. 291-313, 2015.

[60] M. Romano, V. Ricci, A. di Popolo et al., "Helicobacter pylori upregulates expression of epidermal growth factor-related peptides, but inhibits their proliferative effect in MKN 28 gastric mucosal cells," The Journal of Clinical Investigation, vol. 101, no. 8, pp. 1604-1613, 1998.

[61] M. Ratti, A. Lampis, J. C. Hahne, R. Passalacqua, and N. Valeri, "Microsatellite instability in gastric cancer: molecular bases, clinical perspectives, and new treatment approaches," Cellular and Molecular Life Sciences, vol. 75, no. 22, pp. 4151-4162, 2018.

[62] G. Calin, G. N. Ranzani, D. Amadori et al., "Somatic frameshift mutations in the Bloom syndrome BLM gene are frequent in sporadic gastric carcinomas with microsatellite mutator phenotype," BMC Genetics, vol. 2, no. 1, p. 14, 2001.
[63] K. Sakata, G. Tamura, Y. Endoh, K. Ohmura, S. Ogata, and T. Motoyama, "Hypermethylation of the $h M L H 1$ gene promoter in solitary and multiple gastric cancers with microsatellite instability," British Journal of Cancer, vol. 86, no. 4, pp. 564-567, 2002.

[64] S. Richman, "Deficient mismatch repair: read all about it (review)," International Journal of Oncology, vol. 47, no. 4, pp. 1189-1202, 2015.

[65] P. Ye, Y. Shi, and A. Li, "Association between hMLH1 promoter methylation and risk of gastric cancer: a meta-analysis," Frontiers in Physiology, vol. 9, p. 368, 2018.

[66] N. H. Haron, E. A. Mohamad Hanif, M. R. Abdul Manaf et al., "Microsatellite instability and altered expressions of MLH1 and MSH2 in gastric cancer," Asian Pacific Journal of Cancer Prevention, vol. 20, no. 2, pp. 509-517, 2019.

[67] J. C. Santos and M. L. Ribeiro, "Epigenetic regulation of DNA repair machinery in Helicobacter pylori-induced gastric carcinogenesis," World Journal of Gastroenterology, vol. 21, no. 30, pp. 9021-9037, 2015.

[68] D. Nejman, I. Livyatan, G. Fuks et al., "The human tumor microbiome is composed of tumor type-specific intracellular bacteria," Science, vol. 368, no. 6494, pp. 973-980, 2020.

[69] J. Gagnière, J. Raisch, J. Veziant et al., "Gut microbiota imbalance and colorectal cancer," World Journal of Gastroenterology, vol. 22, no. 2, pp. 501-518, 2016.

[70] C. L. Sears and W. S. Garrett, "Microbes, microbiota, and colon cancer," Cell Host \& Microbe, vol. 15, no. 3, pp. 317328, 2014.

[71] K. W. Kinzler and B. Vogelstein, "Lessons from hereditary colorectal cancer," Cell, vol. 87, no. 2, pp. 159-170, 1996.

[72] S. Hwang, M. Jo, J. E. Hong et al., "Zerumbone suppresses enterotoxigenic Bacteroides fragilis infection-induced colonic inflammation through inhibition of NF- $\kappa B$," International Journal of Molecular Sciences, vol. 20, no. 18, p. 4560, 2019.

[73] L. Chung, E. Thiele Orberg, A. L. Geis et al., "Bacteroides fragilis Toxin Coordinates a Pro-carcinogenic Inflammatory Cascade via Targeting of Colonic Epithelial Cells," Cell Host \& Microbe, vol. 23, no. 2, pp. 203-214.e5, 2018.

[74] J. I. Jeon, S. H. Ko, and J. M. Kim, "Intestinal epithelial cells exposed to Bacteroides fragilis enterotoxin regulate NF- $\kappa \mathrm{B}$ activation and inflammatory responses through $\beta$-catenin expression," Infection and Immunity, vol. 87, no. 11, 2019e00312-19.

[75] C. M. Dejea, P. Fathi, J. M. Craig et al., "Patients with familial adenomatous polyposis harbor colonic biofilms containing tumorigenic bacteria," Science, vol. 359, no. 6375, pp. 592597,2018

[76] E. Senkus, S. Kyriakides, S. Ohno et al., "Primary breast cancer: ESMO Clinical Practice Guidelines for diagnosis, treatment and follow-up "," Annals of Oncology, vol. 26, pp. v8-v30, 2015.

[77] "U.S. Breast Cancer Statisticshttps," 2017, March 2019, http:// Breastcancer.org, http://breastcancer.org/symptoms/ understand_bc/statistics.

[78] A. Bleyer and H. G. Welch, "Effect of three decades of screening mammography on breast-cancer incidence," The New England Journal of Medicine, vol. 367, no. 21, pp. 1998-2005, 2012.

[79] E. Mikó, T. Kovács, E. Sebő et al., “Microbiome-microbial metabolome-cancer cell interactions in breast cancerfamiliar, but unexplored," Cell, vol. 8, no. 4, pp. 293-302, 2019. 
[80] C. Xuan, J. M. Shamonki, A. Chung et al., "Microbial dysbiosis is associated with human breast cancer," PLoS One, vol. 9, no. 1, article e83744, 2014.

[81] A. F. Kantor, P. Hartge, R. N. Hoover, A. S. Narayana, J. W. Sullivan, and J. F. Fraumeni Jr., "Urinary tract infection and risk of bladder cancer," American Journal of Epidemiology, vol. 119, no. 4, pp. 510-515, 1984.

[82] J. S. Lehman, Z. Farid, J. H. Smith, S. Bassily, and N. A. ElMasry, "Urinary schistosomiasis in Egypt: clinical, radiological, bacteriological and parasitological correlations," Transactions of the Royal Society of Tropical Medicine and Hygiene, vol. 67, no. 3, pp. 384-399, 1973.

[83] R. M. Hicks, M. M. Ismail, C. L. Walters, P. T. Beecham, M. F. Rabie, and M. A. el Alamy, "Association of bacteriuria and urinary nitrosamine formation with Schistosoma haematobium infection in the Qalyub area of Egypt," Transactions of the Royal Society of Tropical Medicine and Hygiene, vol. 76, no. 4, pp. 519-527, 1982.

[84] S. Calmels, H. Ohshima, and H. Bartsch, "Nitrosamine formation by denitrifying and non-denitrifying bacteria: implication of nitrite reductase and nitrate reductase in nitrosation catalysis," Journal of General Microbiology, vol. 134, no. 1, pp. 221226, 1988.

[85] A. R. Tricker, D. J. Stickler, J. C. Chawla, and R. Preussmann, "Increased urinary nitrosamine excretion in paraplegic patients," Carcinogenesis, vol. 12, no. 5, pp. 943-946, 1991.

[86] D. Umbenhauer, C. P. Wild, R. Montesano et al., "06-Methyldeoxyguanosine in oesophageal DNA among individuals at high risk of oesophageal cancer," International Journal of Cancer, vol. 36, no. 6, pp. 661-665, 1985.

[87] S. H. Kroft and R. Oyasu, "Urinary bladder cancer: mechanisms of development and progression," Laboratory Investigation, vol. 71, no. 2, pp. 158-174, 1994. 